\title{
Factors Affecting Prognosis in Metastatic Colorectal Cancer Patients
}

\author{
Baki Eker $^{1 \& *}$, Ersin Ozaslan ${ }^{2 \&}$, Halit Karaca², Veli Berk², Oktay Bozkurt², \\ Mevlude Inanc ${ }^{2}$, Ayse Ocak Duran², Metin Ozkan²
}

\begin{abstract}
Background: Colorectal cancer (CRC) is a major cause of mortality in developed countries, and it is the third most frequent malignancy in Turkey. There are many biological, genetic, molecular, and tissue-derived prognostic factors for CRCs. In this study, we evaluated prognostic factors in patients who were metastatic at diagnosis or progressed to metastatic disease during follow-up. Patients and Methods: This study included 116 patients with malignancies either in the colon or rectum. Of these, 65 had metastatic disease at diagnosis, and 51 progressed to metastatic disease during the course of the disease. The parameters evaluated were age, gender, comorbidity, performance status and stage of the disease at the beginning, localization, history of surgery, chemotherapy regimen, response to first-line treatment, K-RAS status, site and number of metastases, expression of tumor predictors (CEA, CA19-9), and survival times. A multivariate analysis conducted with factors that considered statistically significant in the univariate analysis. Findings: Median age was $56(32-82)$ years and the male/ female ratio was 80/36. Eleven patients were at stage II, 40 at stage III, and 65 at stage IV at diagnosis. Twenty three patients had tumor in the right colon, 48 in the left colon, and 45 in the rectum. Ninety seven patients were operated, and 27 had surgical metastasectomy. Ninety three patients received targeted therapy. At the end of follow-up, 61 patients had died, and 55 survived. Metastatic period survival times were longer in the adjuvant group, but the difference did not reach the level of statistical significance (adjuvant group: median 29 months,

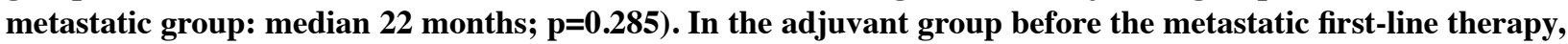
CEA and CA 19-9 levels were significiantly lower compared to the metastatic group $(\mathbf{p}<0.005)$. We also found that patients with elevated tumor predictor (CEA, CA 19-9) levels before the first-line therapy had significiantly poorer prognosis and shorter survival time. Survival was significiantly better with the patients who were younger than 65 years of age, had better initial performance status, a history of primary surgery and metastatectomy, and single site of metastasis. Those who benefitted from the first-line therapy were K-RAS wild type and whose tumor markers (CEA, CA 19-9) were not elevated before the first line therapy. Conclusions: Among the patients with metastatic CRC, those who benefited from first-line therapy, had history of metastasectomy, were K-RAS wild type and had low CA 19-9 levels before the first-line therapy, showed better prognosis independent of other factors.
\end{abstract}

Keywords: Colorectal cancer - prognostic factors - survival - stage at diagnosis

Asian Pac J Cancer Prev, 16 (7), 3015-3021

\section{Introduction}

Colorectal cancers (CRC) are the most frequently encountered cancers of the gastrointestinal system, which is also the third most common cancer type among men following prostate and lung and in women following breast and lung cancer. Tumor predictors are one of the aiding tools used for diagnosis and follow up of reaction to treatment in colorectal cancers. Predictors used in colon cancers are carcino-embryonic antigen (CEA) and carbohydrate antigen (CA) 19-9.

The most important prognostic factor in colorectal cancer is the stage of the disease. However, there are other various biological, genetic, molecular, and tissue-related prognostic factors other than the stage of the diseases. American Pathology Association reported the following conditions proven to have prognostic importance for colorectal cancers: local tumor size indicating the depth of tumor penetration (Chapuis et al., 1985), number of regional lymph nodes (Washington, 2010), presence of mesenteric lymph nodes also described as accumulation of satellite tumors according to 2010 tumor-lymph nodemetastasis (TNM) classification (Edge et al., 2010), vascular invasion (Michelassi et al., 1991), presence of residual tumor following curative treatment (Compton et al., 2000), and pre-operative CEA levels (Park et al., 2009). A recent study reported that while TNM staging was considered the most important prognostic for $\mathrm{CRC}$, it was 
also emphasized that age, preoperative obstruction, CEA levels at time of diagnosis, resection condition, pathologic type, histologic grade, and lymphovascular invasion also had prognostic significance. The initial treatment method for non-metastatic CRC is surgical intervention. While primary tumor resection can be performed in case of complication development in metastatic diseases, survival can be prolonged via metastasectomy performed on resectable metastatic foci (Nordlinger et al., 2009; Schule et al., 2013).

While palliative chemotherapy can enhance survival in metastatic CRC (mCRC), it can also help suppress the symptoms, increase the quality of life, and make potentially resectable isolated liver and lung metastases more suitable for surgery by some reduction in size. There have been more significant advancements in CRC treatment over the past five years as the target-oriented agents introduced to the clinical practice. Bevacizumab, cetuximab and panitumumab (anti-EGFR monoclonal antibody) are the agents proven to have an impact on survival (Hurwitz et al., 2004; Van Cutsem et al., 2009; Kiss et al., 2014). At the end of the long-term follow-up, it was observed that the difference between progression-free survival (PFS) and overall survival (OS) of KRAS wild type and mutant patients continuously increased, and the OS difference had reached level of statistical significance (Maughan et al., 2009; Bokemeyer et al., 2012). K-RAS mutation status is an important predictive factor for the treatment response to anti-EGFR monoclonal antibodies in CRC. When treating K-RAS 'wild type' CRC patients, cetuximab and panitumumab therapy is recommended.

\section{Materials and Methods}

There were 116 metastatic colon or rectal cancer (mCRC) patients included in this study who were being treated between 2001 and 2011 at the Department of Medical Oncology, Erciyes University Medical School. Among these patients, 51 were at stage II-III at the time of diagnosis then they had progressed to stage IV during follow-up (Group 1, adjuvant group). The remaining 65 were metastatic (stage IV) at the time of diagnosis (Group 2 , metastatic group).

Variables such as age, gender, comorbidity, performance status and disease stage at the time of diagnosis, tumor localization, surgical history, tumor predictors (CEA, CA 19-9) at the time of diagnosis, pre-treatment and posttreatment levels, number of cures at each line of treatment, durations of disease free survival (DFS), overall survival (OS) and progression-free survival (PFS), K-RAS status, treatment regimens, and biological agent (s) used and their impact on survival and recurrence were evaluated for each patient. For Group 1, the pathologic TNM staging at the time of diagnosis, whether they received adjuvant therapy or not, and their adjuvant therapy regimens were also evaluated.

The time from the beginning of the treatment to the progression in Group 1 was calculated as DFS, time from the beginning of the treatment after the metastasis detection until the progression as PFS, and the time until death as OS.
CEA and CA 19-9 measurements were made with serum samples using ADVIA Centaur ${ }^{\circledR}$ CEA and CA 19-9 (Bayer Diagnostics, Tarrytown, NY) kits via twostep chemiluminometric measurement technique. The reference ranges based on these were $0-5 \mathrm{ng} / \mathrm{mL}$ for CEA and $0-35 \mathrm{U} / \mathrm{mL}$ for CA 19-9. Values above the upper limit were considered as positive.

The groups were compared in terms of demographic data and factors impacting prognosis and survival.

Frequency, ratio, mean, and standard deviation values were used for descriptive statistics of data. The distribution pattern of the variables was checked with Kolmogorov Smirnov test. Analysis of variance (Tukey test), independent samples t-test, and Mann Whitney U test were used in the analyses. Analyses of proportional data were conducted via chi-square test. The effects of prognostic factors on survival were examined with both univariate and multivariate analyses. Kaplan-Meier method was used for univariate survival analyses and within group comparisons were done via log-rank test. Cox regression test's backward Wald method was used for multivariate analyses. Level of significance was set at $\mathrm{p}<0.05$ within a $95 \%$ confidence interval (CI). SPSS 20.0 software was used for analyses.

\section{Results}

Median age of the 116 patients included in this study was 56 (range: $32-82$ ) years. Median time from diagnosis to treatment among patients in the adjuvant group was 2 (range: 1-10) months. Mean CEA value among patients prior to the initial metastatic treatment was $250.2 \pm 1057.1$ $\mathrm{ng} / \mathrm{mL}$ and 68 patients $(59.6 \%)$ had high CEA values. Mean CA 19-9 value was $1029.2 \pm 6658.9 \mathrm{U} / \mathrm{mL}$ and 54 patients $(47.4 \%)$ had high CA $19-9$ values. In $37 \%$ of patients both of them (CEA and CA 19-9) were high before treatment.

When the first-line metastatic treatment of patients was screened, it was observed that 41 patients $(35.3 \%)$ received oxaliplatin-based regimens and 72 patients $(62 \%)$ received irinotecan-based regimens. Additionally, 31 (26.7\%) of the patients received regimens which include oral fluoropyrimidine. Again as first-line treatment, 73 patients $(62.9 \%)$ used regimens including bevacizumab.

Regarding the second-line treatments, 14 of the $(21 \%)$ 66 patients were on regimens including cetuximab. When all treatments were considered, 23 patients (19.8\%) did not use any biologic agents, 19 (16.4\%) received bevacizumab and cetuximab consecutively. Only one of these treatments (bevacizumab or cetuximab) was used in $74(63.8 \%)$ patients.

As displayed in Table 1, mean CEA and CA 19-9 values prior to the first-line metastatic treatment were significantly lower in the adjuvant group compared to the metastatic group. The number of patients with high CEA and CA 19-9 values, prior to the treatment, was also significantly higher in the metastatic group $(\mathrm{p}<0.005)$.

Median age of the patients in the adjuvant $(n=51)$ and metastatic groups $(n=65)$ was 55 and 57 years, respectively ( $\mathrm{p}=0.059)$. Male patients constituted $70.6 \%$ of the patients in the adjuvant group and $67.7 \%$ of the 


\begin{tabular}{|c|c|c|c|c|}
\hline & & $\begin{array}{c}\text { Adjuvant Group } \\
\mathrm{n}(\%)\end{array}$ & $\begin{array}{c}\text { Metastatic Group } \\
\mathrm{n}(\%)\end{array}$ & $\mathrm{p}$ \\
\hline Baseline CEA (mean \pm ss) & & $11.6 \pm 29.9$ & & \\
\hline Baseline CA 19-9(mean \pm ss $)$ & & $25.2 \pm 57.4$ & & \\
\hline Pretreatment CEA(mean \pm ss $)$ & & $23.0 \pm 44.6$ & $434 \pm 1399.3$ & 0.003 \\
\hline Pretreatment CA 19-9(mean \pm ss $)$ & & $49.2 \pm 155.3$ & $1822.6 \pm 8908.8$ & $<0.001$ \\
\hline \multirow[t]{2}{*}{ Pre-treatment CEA(ng/mL) } & high & $23(45.1)$ & $45(71.4)$ & \\
\hline & normal & $28(54.9)$ & $18(28.6)$ & 0.004 \\
\hline \multirow[t]{2}{*}{ Pre-treatment CA 19-9(U/mL) } & high & $13(25.5)$ & $41(65.1)$ & \\
\hline & normal & $38(74.5)$ & $22(34.9)$ & $<0.001$ \\
\hline \multirow[t]{3}{*}{ Baseline PS score } & 0 & $36(70.5)$ & $29(44.6)$ & 0.012 \\
\hline & 1 & $14(27.4)$ & $29(44.6)$ & \\
\hline & 2 & $1(2.1)$ & $7(10.8)$ & \\
\hline \multirow[t]{2}{*}{ Comorbidity } & Yes & $7(13.7)$ & $39(60)$ & 0.001 \\
\hline & No & $44(86.3)$ & $26(40)$ & \\
\hline Patients who undergone primary surgery & & $51(100)$ & $46(70.8)$ & 0.001 \\
\hline Patients who undergone metastasectomy & & $21(41.2)$ & $6(9.2)$ & 0.001 \\
\hline \multirow[t]{4}{*}{ Site of metastasis } & Liver & $15(29.5)$ & $34(52.3)$ & \\
\hline & Lung & $12(23.5)$ & $1(1.5)$ & 0.001 \\
\hline & Multiple & $12(23.5)$ & $2(3)$ & \\
\hline & Other & $12(23.5)$ & $28(43.2)$ & \\
\hline \multirow[t]{2}{*}{ Receiving Bevacizumab at first-line therapy } & Yes & $40(78.4)$ & $33(50.8)$ & 0.002 \\
\hline & No & $11(21.6)$ & $32(49.2)$ & \\
\hline \multirow[t]{2}{*}{ First-line therapy regimen } & Oxaliplatin-based & $8(15.6)$ & $33(50.8)$ & 0.001 \\
\hline & Irinotecan- based & $40(78.4)$ & $32(49.2)$ & \\
\hline Median PFS1(months) (95\% CI) & & $11(6.6-15.3)$ & $7.5(5.9-9)$ & 0.258 \\
\hline Median PFS2(months) (95\% CI) & & $4.5(2.6-6.3)$ & $6(4.4-7.5)$ & 0.836 \\
\hline Median OS (months) $(95 \% \mathrm{CI})$ & & $29(14.3-43.7)$ & $22(15.5-28.5)$ & 0.285 \\
\hline
\end{tabular}

Table 2. Effects of Other Factors on Overall Survival (OS)

\begin{tabular}{|c|c|c|}
\hline \multirow[b]{2}{*}{ Variable (n) } & \multicolumn{2}{|c|}{ OS (months) } \\
\hline & Median $(95 \% \mathrm{CI})$ & $\mathrm{p}$ \\
\hline \multicolumn{3}{|l|}{ Baseline PS score } \\
\hline $0(n=65)$ & $33(18.8-47.1)$ & \\
\hline $1(n=43)$ & $21(14.6-27.3)$ & 0.001 \\
\hline $2(n=8)$ & $8(6.1-9.8)$ & \\
\hline \multicolumn{3}{|l|}{ Metastasectomy } \\
\hline Yes $(n=27)$ & $45(29.2-60.7)$ & \\
\hline No $(n=89)$ & $22(18.5-25.4)$ & 0.001 \\
\hline \multicolumn{3}{|l|}{ Biologic Agents } \\
\hline Received $(n=74)$ & $24(17.9-30.1)$ & \\
\hline $\begin{array}{l}\text { Received both of } \\
\text { them }(n=19)\end{array}$ & $45(21.1-68.8)$ & 0.063 \\
\hline Not received $(n=23)$ & $18(2.4-33.5)$ & \\
\hline \multicolumn{3}{|c|}{ Clinical benefit from firstline therapy } \\
\hline Yes $(n=43)$ & $41(14.8-67.1)$ & 0.001 \\
\hline No $(n=73)$ & $18(7.3-28.6)$ & \\
\hline \multicolumn{3}{|l|}{ Site of metastasis } \\
\hline Single $(\mathrm{n}=80)$ & $30(19.6-40.3)$ & 0.005 \\
\hline Multiple $(n=36)$ & $18(10.8-25.1)$ & \\
\hline
\end{tabular}

patients in the metastatic group ( $\mathrm{p}=0.074)$. Baseline PS scores were significantly better and comorbidity rates were significantly lower in adjuvant group.

As displayed in the Table 1, the metastatic period survival of the adjuvant group (OS) was longer than that of the metastatic group (29 and 22 months, respectively) but this difference was not statistically significant ( $\mathrm{p}=0.285$ ). The progression-free survival during first-line and secondline treatments was similar across groups.

In this study, 37 patients in the adjuvant group $(n=51)$ received FOLFOX regimen as adjuvant treatment and 12 received fluorouracil+folinic acid (FUFA), while 2 patients were not administered adjuvant therapy. Among the patients with positive and negative K-RAS mutation, the DFS was 13 and 17 months, respectively. However, this difference was not statistically significant ( $\mathrm{p}=0.620)$. Other factors affecting OS during metastatic periods are presented in Table 2.

According to this evaluation, survival is significantly longer among those who had better baseline performance status, undergone metastasectomy, and benefitted from first-line treatment (Figure 1). Use of biologic agents was also observed to prolong survival but this difference was not significant but it was so close to significance $(\mathrm{p}=0.063)$.

The factors proven to have significant impact in univariate analyses were age, high CEA and CA 19-9 values prior to the first-line treatment, having benefit from the first-line treatment or not, presence of K-RAS mutation, history of primary surgery, history of metastasis

Table 3. Independent Prognostic Factors for Patients with mCRC upon Multivariate Analysis

\begin{tabular}{lccc}
\hline Multivariate Analysis & Relative Risk & CI (95\%) & $\mathrm{p}$ \\
\hline Number of cures that received at first-line therapy & 0.682 & $0.593-0.785$ & 0.001 \\
Metastasectomy & 3.958 & $1.654-9.471$ & 0.002 \\
K-RAS state & 0.301 & $0.107-0.843$ & 0.022 \\
CA 19-9 level prior to metastatic first-line therapy & 2.067 & $1.177-3.627$ & 0.011 \\
\hline
\end{tabular}




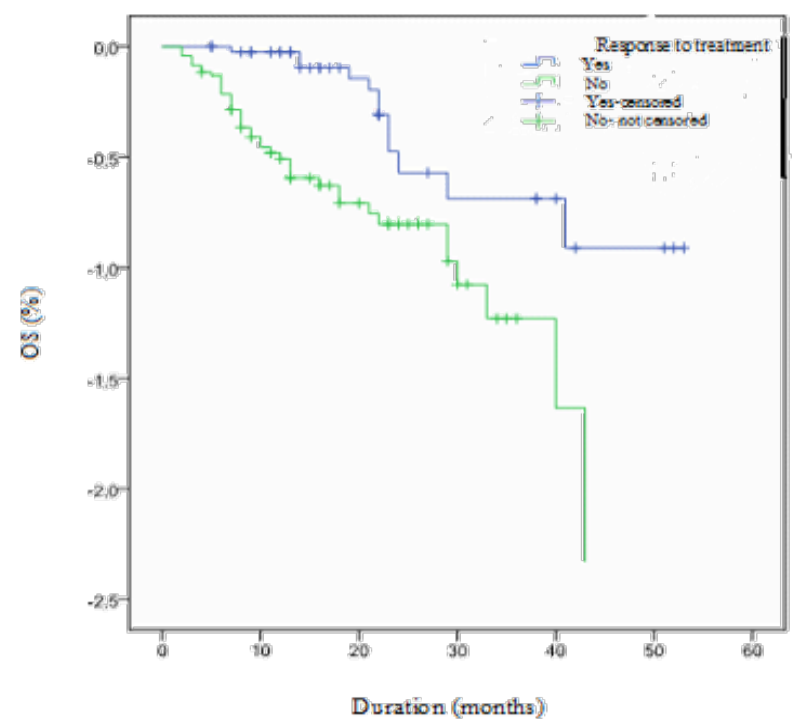

Figure 1. Metastatic First-line Therapy Response-OS Curves

surgery; number of cures in the first-line treatment, and baseline performance status. These significant factors were included in the multivariate model where number of cures in first-line treatment, history of metastasis surgery, K-RAS status, and high CA 19-9 levels prior to the firstline treatment were the factors that remained significant. These factors were considered independent prognostic factors for patients with metastatic CRC. Table 3 presents a summary of these factors.

\section{Discussion}

Prior to choosing the most appropriate treatment approach for CRC patients, it is essential to clearly identify the prognostic factors. In this study, we aimed at identifying the prognostic factors for mCRC. Moreover, we compared the clinical characteristics and prognostic factors among patients who were stage II or III initially and had distant metastasis afterwards and patients who were stage IV at the time of diagnosis. When the metastasis locations were investigated, the most common metastasis location was liver, in accordance with medical literature (42\%). The most common metastasis location was still liver when it was considered by groups. In a prior study by Patanaphan et al. liver was reported as the most common location of metastasis in both patients who were metastatic at the time of diagnosis and those who became metastatic afterwards (Patanaphan et al., 1993). The same study also reported that the metastasis location that most negatively affected survival time was brain (5.5 months), followed by liver ( 9 months). OS was reported as 10 months for those with multiple metastases (Patanaphan et al., 1993). There are other studies that failed to identify an association between the location of metastasis and survival (Wang et al., 2002; Gonsalves et al., 2012). In a study by Khattak et al., the metastasis locations and survival durations were compared and reported that the longest survival was observed among those with only lung metastasis (41 months) and that the shortest was observed among those with isolated bone and brain metastases (5.1 and
5.7 months; respectively) (Khattak et al., 2012). Again, in another study, both the location and the number of extra hepatic metastases were reported to be associated with poor prognosis (Pulitano et al., 2011). In our study, no significant association was identified between metastasis location and survival. However, multiple metastatic cases were observed to have significantly shorter OS compared to those with single metastasis (18 months and 30 months, respectively; $\mathrm{p}=0.005$ ). The most common location for metastasis was still liver in both groups in our study. However, considering the ratios, while liver metastasis rate was $29 \%$ in the adjuvant group, it was $52 \%$ in the metastatic group and the difference was statistically significant.

There are numerous studies in medical literature that investigated tumor predictors, especially CEA in CRC. Some studies reported that the pre-operative CEA level was an independent prognostic factor affecting the CRC survival (Sun et al., 2009; Chew et al., 2012; He et al., 2014). A study reported that the survival decreased among patients with high pre-operative CEA levels and that the pre-operative CEA was correlated with tumor, tumor invasion depth, lymph node condition, stage, and post-operative recurrence (Wang et al., 2007). Many studies reported that when evaluated together, CEA and CA 19-9 levels increase sensitivity and specificity and that high levels indicate poor prognosis, where PFS and OS decreased significantly (Dixon et al., 2003; Wang et al., 2007; Sun et al., 2009; Dong et al., 2013; Sisik et al., 2013). Some studies conducted on CA 19-9 reported that it was of greater sensitivity than CEA in case of advanced stage diseases (Wang et al., 2002; Mourtzikou et al., 2012). On the other hand, there are studies reporting that CEA had greater sensitivity (Chapuis et al., 1985; Von Kleist, 1986; Filella et al., 1992). Xavier et al.'s prior study reported that while CEA was a more sensitive measure than CA 19-9, it did not have prognostic significance and that CA 19-9 was the only factor independently associated with PFS (Filella et al., 1992). Kim et al.'s study reported that CEA was useful only in the follow-up of the cases with high CEA levels initially and that its sensitivity and diagnostic accuracy was low in cases with normal initial CEA levels (Kim et al., 2013). In Wang et al.'s study, CA 19-9 was found to be the only tumor predictor, independently associated with prognosis in CRC cases. In our study, in line with these findings, high levels of the tumor predictors CEA and CA 19-9 prior to treatment were significantly associated with poor diagnosis. Survival of such patients significantly decreased. Moreover, the mean pre-treatment levels of CEA and CA 19-9 were significantly lower in the adjuvant group, compared to the metastatic group; while the mean CEA and CA 19-9 levels were 23 and 49.2, respectively, in the adjuvant group and 434 and 1822, respectively, in the metastatic group ( $\mathrm{p}=0.003, \mathrm{p}=0.001$ ). This was most likely due to the continuous follow-up of the patients in the adjuvant group, and the fact that they were diagnosed at a time of low tumor load. Despite this fact, no significant difference was observed between the adjuvant and metastatic groups in terms of OS ( 29 and 22 months, respectively). The failure to detect significance in 
this difference may be due to the small sample size. The significant effect of CEA, one of the tumor predictors with an impact on survival observed in the univariate analysis diminished in multivariate analysis. CA 19-9's significant effect, on the other hand, was sustained in multivariate analysis as well. According to this finding, in accordance with Wang and Xavier's findings, CA 19-9 was identified as a significant independent prognostic predictor in our study (Filella et al., 1992; Wang et al., 2002). Also, in a recent study it was demonstrated that CA 19-9 was superior to CEA in post-operative follow-up (Samowitz et al., 2007).

Performing primary tumor resection in CRC cases when possible significantly increases survival. Primary tumor resection is recommended even for stage IV patients (Samowitz et al., 2007; Chew et al., 2012; Padman et al., 2013; Yoon et al., 2014). In a study by Moghimi-Dehkordi et al.it was determined that a surgical intervention targeting primary tumors was significantly associated with survival in univariate analysis but this significance diminished in multivariate analysis, leading to not being considered an independent prognostic factor. Consistent with findings of this study, our study findings have also demonstrated that when all patients were considered, OS and PFS were significantly prolonged among patients who had undergone primary surgery $(\mathrm{p}=0.001 ; \mathrm{p}=0.016)$. However, surgery targeting primary tumors lost its significant impact in multivariate analyses. In our study, OS was determined to have been significantly prolonged among patients who had undergone metastasis surgery, compared to those who had not (45 vs 22 months, $\mathrm{p}=0.001)$; and this effect had continued to be significant in multivariate analyses as well. Additionally, the ratio of patients who had undergone metastasis surgery was significantly higher in the adjuvant group compared to the metastatic group (41\% vs 9\%, $\mathrm{p}=0.001$ ). These findings are consistent with many studies in medical literature, demonstrating that metastasectomy improves survival. These studies reported that liver and lung metastasectomy in CRC cases significantly improved survival (Tournigand et al., 2004; Inoue et al., 2004; Cokmert et al., 2014). Additionally, the higher number of patients who had undergone metastasis surgery in the adjuvant group may be due to the fact that these patients were followed-up and that their metastases had been detected in early stages. Christos et al's study, one of the most important studies on K-RAS mutations in CRCs up-to-date, reported significant improvements in PFS and OS among "wild type" negative K-RAS mutation patients receiving cetuximab monotherapy compared to those receiving supplemental therapy. No significant association was observed between survival and K-RAS condition of those receiving supplemental therapy only. In other words, it was determined that K-RAS's impact on survival depends on the therapy (Christos et al., 2008). In parallel with the data reported by our department beforehand, in smaller-scale retrospective studies, survival was found to be longer among K-RAS "wild type" CRC patients (Karagkounis et al., 2013; Bozkurt et al., 2014; Cvetanovic et al., 2014). In our study, survival was significantly longer among K-RAS "wild type" CRC patients (45 vs 24 months) and this difference was significant in multivariate analyses as well. However, this improvement we detected in survival was not independent of the treatment because almost all of the K-RAS "wild type" patients were on cetuximab therapy. No significant difference was observed between K-RAS "wild type" and mutant patients in terms of PFS in our study but the difference was close to reach significance. This finding, on the other hand, was independent of the treatment because no patient received cetuximab in the first-line therapy (10 and 7.5 months respectively, $\mathrm{p}=0.059$ ). Again no significant association was observed between DFS survival and K-RAS condition in the adjuvant group. Therefore, our findings on K-RAS condition are consistent with those of Christos et al.'s findings (2008). There have been more significant advancements in survival over the past five years as target-oriented agents were used widely in metastatic CRCs. Anti-EGFR monoclonal antibodies are agents proven to have a positive impact on survival. It was reported that, in mCRC patients who have not treated before, adding bevacizumab to FOLFIRI regimen prolonged OS for 4.7 months and enhanced treatment response ratios about $\% 10$. In mCRC patients, who have treated before, adding bevacizumab to oxaliplatin-based therapy achieved significantly prolonged survival rates, however, in patients who received this combination in first-line therapy, there was any significant enhancement in survival (Giantonio et al., 2007; Saltz et al., 2008).

In our study, in patients who received bevacizumab during first-line therapy, there was not a significant enhancement in terms of OS and PFS. Our study patients were separated in to three groups as "received any biologic agents", "received one of the biologic agents" and "received bevacizumab and cetuximab consequently". OS was 18, 24 and 45 months for these groups, respectively. But this difference between groups was not statistically significant however it was close to reach significance $(\mathrm{p}=0.063)$. Consequently, in our study we could not demonstrate a significant advance in OS with biologic agents; however, it is likely to be significant with a larger population.

In conclusion, consistent with literature findings, this study has determined that among metastatic CRC patients, those who benefit from first-line therapy, have had an opportunity to undergo metastasis surgery, have negative K-RAS condition, and have low CA-19-9 levels prior to first-line therapy have better prognosis independent of other factors.

\section{References}

Bokemeyer C, Van Cutsem E, Rougier P, et al (2012). A metaanalysis of the CRYSTAL and OPUS studies combining cetuximab with chemotherapy as 1st-line treatment for patients with metastatic colorectal cancer: results according to KRAS and BRAF mutation status. Eur J Cancer, 48, 1466-75.

Bozkurt O, Inanc M, Turkmen E, et al (2014). Clinicopathological characteristics and prognosis of patients according to recurrence time after curative resection for colorectal cancer. Asian Pac J Cancer Prev, 15, 9277-81.

Chapuis PH, Dent OF, Fisher R, et al (1985). A multivariate analysis of clinical and pathological variables in prognosis 
after resection of large bowel cancer. Br J Surg, 72, 698-702.

Chew MH, Teo JY, Kabir T, et al (2012). Stage IV colorectal cancers: an analysis of factors predicting outcome and survival in 728 cases. J Gastrointest Surg, 16, 603-12.

Christos S, Karapetis, ShirinKhambata-Ford, et al (2008). K-ras mutations and benefit from cetuximab in advanced colorectal cancer. N Engl J Med, 359, 1757-65.

Cokmert S, Ellidokuz H, Demir L, et al (2014). Survival outcomes of liver metastasectomy in colorectal cancer cases: a single-center analysis in Turkey. Asian Pac J Cancer Prev, 15, 5195-200.

Compton CC, Fielding LP, Burqart LJ, et al (2000). Prognostic factors in colorectal cancer. college of American Pathologists consensus statement 1999. Arch Pathol Lab Med, 124, 979-94.

Cvetanovic A, Vrbic S, Filipovic S, et al (2014). Clinical benefit of cetuximab and prognostic value of cetuximab-related skin toxicity in metastatic colorectal cancer: a single in stitution analysis. J BUON, 19, 83-90.

Dixon MR, Haukoos JS, Udani SM, et al (2003). Carcino embryonic antigen and albumin predict survival in patients with advanced colon and rectal cancer. Arch Surg, 138, 962-6.

Dong H, Tong J, Li LH, et al (2013). Serum carbonhydrate antigen 19-9 as an indicator of liver metastasis in colorectal carcinoma cases. Asian Pac J Cancer Prev, 14, 909-13.

Edge SB, CC Compton (2010). The American joint committee on cancer: the $7^{\text {th }}$ edition of the AJCC cancer staging manual and the future of TNM. Ann Surg Oncol, 17, 1471-4.

Filella X, Molina R, Grau JJ, et al (1992). Prognostic value of CA 19-9 levels in colorectal cancer. Ann Surg, 216, 55-9.

Giantonio BJ, Catalano PJ, Meropol NJ, et al (2007). Bevacizumab in combination with oxaliplatin, fluorouracil, and leucovorin (FOLFOX4) for previously treated metastatic colorectal cancer: results from the Eastern cooperative oncology group study E3200. J Clin Oncol, 25, 1539-44.

Gonsalves WI, Wolpert J, Tashi T, et al (2012). Assessment of prognostic factors after primary tumor resection in metastatic colon cancer patients: a veteran's affairs central cancer registry (VACCR) analysis, 1995-2008. J Surg Oncol, 106, 486-90.

He WZ, Jiang C, Yin CX, et al (2014). Prognostic model built on blood-based biomarkers in patients with metastatic colorectal cancer. Asian Pac J Cancer Prev, 15, 7327-31.

Hurwitz H, Fehrenbacher L, Novotny W, et al (2004). Bevacizumab plus irinotecan, fluorouracil, and leucovorin for metastatic colorectal cancer. N Engl J Med, 350, 2335-42.

Inoue M, Ohta M, Iuchi K, et al (2004). Benefits of surgery for patients with pulmonary metastases from colorectal carcinoma. Ann Thorac Surg, 78, 238-44.

Karagkounis G, Torbenson MS, Daniel HD, et al (2013). Incidence and prognostic impact of KRAS and BRAF mutation in patients undergoing liver surgery for colorectal metastases. Cancer, 119, 4137-44.

Khattak MA, Martin HL, Beeke C, et al (2012). Survival differences in patients with metastatic colorectal cancer and with single site metastatic disease at initial presentation: results from South Australian clinical registry for advanced colorectal cancer. Clin Colorectal Cancer, 11, 247-54.

Kim G, Jung EJ, Ryu CG, et al (2013). Usefulness of carcino embryonic antigen for monitoring tumor progression during palliative chemotherapy in metastatic colorectal cancer. Yonsei Med J, 54, 116-22.

Kiss I, Bortlicek Z, Melichar B, et al (2014). Efficacy and toxicity of bevacizumab on combination with chemotherapy in different lines of treatment for metastatic colorectal carcinoma. Anticancer Res, 34, 949-54.
Maughan T, Adams RA, Smith CG, et al (2009). Addition of cetuximab to oxaliplatin-based combination chemotherapy in patients with KRAS wild-type advanced colorectal cancer: a randomised superiority trial (MRC COIN). Eur J Cancer, 7, 6LBA (abstr).

Michelassi F, Ayala JJ, Balestracci T, et al (1991). Verification of a new clinico pathologic staging system for colorectal adeno carcinoma. Ann Surg, 214, 11-8.

Mourtzikou A, Stamouli M, Kroupis C, et al (2012). Evaluation of carcino embryonic antigen (CEA), epidermal growth factor receptor (EGFR), epithelial cell adhesion molecule EpCAM (GA733-2), and carbonhydrate antigen 19-9 (CA 19-9) levels in colorectal cancer patients and correlation with clinicopathological characteristics. Clin Lab, 58, 441-8.

Nordlinger B, Van Cutsem E, Gruenberger T, et al (2009). Combination of surgery and chemotherapy and the role of targeted agents in the treatment of patients with colorectal liver metastases: recommendations from an expert panel. Ann Oncol, 20, 985-92.

Padman S, Padbury R, Beeke C, et al (2013). Liver only metastatic disease in patients with metastatic colorectal cancer: impact of surgery and chemotherapy. Acta Oncol, 52, 1699-706.

Park IJ, Choi GS, Lim KH, et al (2009). Serum carcino embryonic antigen monitoring after curative resection for colorectal cancer: clinical significance of the preoperative level. Ann Surg Oncol, 16, 3087-93.

Patanaphan V, Salazar OM (1993). Colorectal cancer; metastatic patterns and prognosis. South Med J, 86, 38-41.

Pulitanò C, Bodingbauer M, Aldrighetti L, et al (2011). Liver resection for colorectal metastases in presence of extrahepatic disease: results from an international multiinstitutional analysis. Ann Surg Oncol, 18, 1380-8.

Saltz LB, Clarke S, az-Rubio E, et al (2008). Bevacizumab in combination with oxaliplatin-based chemotherapy as firstline therapy in metastatic colorectal cancer: a randomized phase III study. J Clin Oncol, 26, 2013-19.

Samowitz WS, Slattery ML, Sweeney C, et al (2007). APC mutations and other genetic and epigenetic changes in colon cancer. Mol Cancer Res, 5, 165-70.

Schüle S, Dittmar Y, Knosel T, et al (2013). Long-term results and prognostic factors after resection of hepatic and pulmonary metastases of colorectal cancer. Int J Colorectal Dis, $\mathbf{2 8}$, 537-45.

Sisik A, Kaya M, Bas G, et al (2013). CEA and CA 19-9 are still valuable markers for the prognosis of colorectal and gastric cancer patients. Asian Pac J Cancer Prev, 14, 4289-94.

Sun LC, Chu KS, Cheng SC, et al (2009). Preoperative serum carcino embryonic antigen, albumin and age are supplementary to UICC staging systems in predicting survival for colorectal cancer patients under going surgical treatment. BMC Cancer, 9, 288.

Tournigand C, Andre T, Achille E, et al (2004). FOLFIRI followed by FOLFOX6 or the reverse sequence in advanced colorectal cancer: a randomized GERCOR study. J Clin Oncol, 22, 229-37.

Van Cutsem E, Kohne CH, Hitre E, et al (2009). Cetuximab and chemotherapy as initial treatment for metastatic colorectal cancer. $N$ Engl J Med, 360, 1408-17.

Von Kleist S (1986). The clinical value of the tumor markers CA 19.9 and carcino embryonic antigen (CEA) in colorectal carcinomas: a critical comparison. Int J Biol Markers, 1, 3-8.

Wang WS, Lin JK, Chiou TJ, et al (2002). CA19-9 as the most significant prognostic indicator of metastatic colorectal cancer. Hepatogastroenterology, 49, 160-4.

Wang JY, Lu CY, Chu KS, et al (2007). Prognostic significance of pre- and postoperative serum carcinoembryonic antigen 
levels in patients with colorectal cancer. Eur Surg Res, 39, 245-50.

Washington $\mathrm{K}$ (2010). $7^{\text {th }}$ edition of the AJCC cancer staging manual: stomach. Ann Surg Oncol, 17, 3077-9.

Yoon YS, Kim CW, Lim SB, et al (2014). Palliative surgery in patients with unresectable colorectal liver metastases: a propensity score matching analysis. J Surg Oncol, 109, $239-44$. 